\title{
The Gigaton Volume Detector in Lake Baikal
}

\section{A. Avrorin ${ }^{\mathrm{a}}$, V. Aynutdinov ${ }^{\mathrm{a}, *}$, I. Belolaptikov $^{\mathrm{d}}, \mathrm{S}$. Berezhnev ${ }^{\mathrm{c}}$, D. Bogorodsky $^{\mathrm{b}}$, N. Budnev ${ }^{\mathrm{b}}$, I. Danilchenko ${ }^{a}$, G. Domogatsky ${ }^{a}$, A. Doroshenko ${ }^{a}, A$. Dyachok ${ }^{b}, Z^{2}$. Dzhilkibaev ${ }^{a}$, G. Ermakov $^{c}$,}

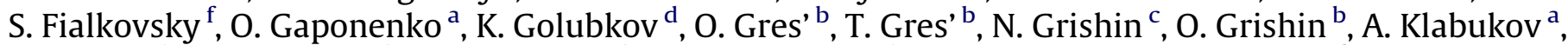
A. Klimov ${ }^{\text {, }}$, A. Kochanov ${ }^{\text {b }}$, K. Konischev ${ }^{\text {d }}$, A. Korobchenko ${ }^{\text {b }}$, A. Koshechkin ${ }^{\text {a }}$, V. Kulepov ${ }^{f}$, D. Kuleshov $^{\text {a }}$, L. Kuzmichev ${ }^{\text {c }}$, V. Lyashuk ${ }^{a}$, E. Middell ${ }^{\text {, }}$, S. Mikheyev ${ }^{a}$, M. Milenin ${ }^{\text {, }}$, R. Mirgazov ${ }^{\text {, }}$, E. Osipova ${ }^{\text {, }}$, A. Pan'kov ${ }^{b}$, L. Pan'kov $^{b}$, A. Panfilov ${ }^{a}$, A. Perevalov ${ }^{b}$, D. Petukhov ${ }^{a}$, E. Pliskovsky ${ }^{\text {d }}$, V. Poleschuk ${ }^{a}$,

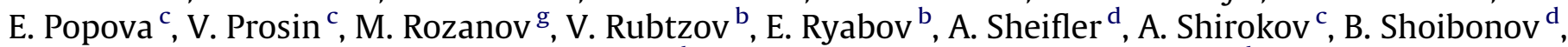
Ch. Spiering ${ }^{\text {e }}$, O. Suvorova ${ }^{\mathrm{a}}$, B. Tarashansky ${ }^{\mathrm{b}}$, R. Wischnewski ${ }^{\mathrm{e}}$, A. Zagorodnikov ${ }^{\mathrm{b}}$, V. Zhukov $^{\mathrm{a}}$, A. Yagunov ${ }^{\mathrm{b}}$

\footnotetext{
a Institute for Nuclear Research, 60th October Anniversary prospect 7a, Moscow 117132, Russia

b Irkutsk State University, Irkutsk, Russia

' Skobeltsyn Institute of Nuclear Physics MSU, Moscow, Russia

d Joint Institute for Nuclear Research, Dubna, Russia

e DESY, Zeuthen, Germany

${ }^{\mathrm{f}}$ Nizhni Novgorod State Technical University, Nizhni Novgorod, Russia

${ }^{g}$ St. Petersburg State Marine University, St. Petersburg, Russia

${ }^{\text {h }}$ Kurchatov Institute, Moscow, Russia
}

\section{A R T I C L E I N F O}

Available online 8 October 2010

Keywords:

Neutrino telescopes

Baikal

\begin{abstract}
A B S T R A C T
The objective of the Baikal Project is the creation of a kilometer-scale high-energy neutrino observatory: the Gigaton Volume Detector (GVD) in Lake Baikal. Basic elements of the GVD - new optical modules, FADC readout units, and underwater communication systems - were investigated and tested in Lake Baikal with prototype strings in 2008-2010. We describe the results of prototype strings operation and review the preliminary design and expected sensitivity of the GVD telescope.
\end{abstract}

c 2010 Elsevier B.V. All rights reserved.

\section{Introduction}

The Baikal collaboration follows for several years a $R \& D$ program for a Gigaton Volume Detector (GVD) in Lake Baikal. The GVD will be a kilometer-scale high-energy neutrino observatory [1]. The main scientific goal of GVD is to map the high-energy neutrino sky in the Southern Hemisphere, including the region of the galactic centre. Other topics include the indirect search for dark matter by searching for neutrinos produced in WIMP annihilation in the sun or at the centre of the Earth. GVD can also be used to search for exotic particles like magnetic monopoles, super-symmetric Q-balls, or nuclearites. In this paper we review the $R \& D$ activities towards a $\mathrm{km}^{3}$-scale Baikal telescope.

\section{Baikal site}

The GVD detector will be located in the southern basin of Lake Baikal nearby to the NT200+ telescope [2-6]. The geographical coordinates of the detector site are $51^{\circ} 50^{\prime} \mathrm{N}$ and $104^{\circ} 20^{\prime} \mathrm{E}$. Since the slope of the shore bottom relief is rather steep, the telescope can be arranged comparatively near the shore, the distance being 4-5 km. The depth of the lake is about $1400 \mathrm{~m}$ at this place. Water currents at the detector site at the depth of about $1 \mathrm{~km}$ is as low as $2 \mathrm{~cm} \mathrm{~s}^{-1}$. The Baikal water is characterized by an absorption length of about $20-25 \mathrm{~m}$ and a scattering length of $30-50 \mathrm{~m}$. Water luminescence is low at the detector site. Rate of light pulses from ${ }^{40} \mathrm{~K}$ decays is negligible.

\section{Design and data acquisition system}

The basic approach for designing the Baikal $\mathrm{km}^{3}$-detector GVD is the same as for NT200. The GVD will consist of strings of optical modules that will be grouped in clusters. This approach provides
* Corresponding author. Tel.: +7 49512335 08; fax: +7 4997839298 .

E-mail address: aynutdin@yandex.ru (V. Aynutdinov). 
a relatively flexible structure that allows for a rearrangement of the main building blocks (clusters), to adapt to requirements of new scientific goals, if necessary. Furthermore, a conventional string structure is an optimum for telescope deployment from the natural ice platform of Lake Baikal. Each string includes a chain of optical modules (OM), spaced uniformly from a depth of 900 to about $1250 \mathrm{~m}$. Each OM contains a photomultiplier tube (PMT), which detects the Cherenkov light produced by relativistic charged particles passing through the water. The information from the ensemble of OMs allows reconstruction of event topology and energy. The photomultiplier R7081HQE was primarily selected as a light sensor for the OMs. This PMT has a hemispherical photocathode with diameter $10 \mathrm{in}$. and quantum efficiency of about 35\%. The construction, functional scheme, and principle of operation of optical module electronics is presented in detail in Refs. [7,8].

Optical modules on the string are grouped into sections. A section is the basic detection unit of GVD [8]. It consists of 12 OMs, the service module (SM), and the electronics unit (BEG) with analog-digital converters on the basis of $200 \mathrm{MHz}$ FADCs. Analog signals from the OMs are transmitted to the BEG through coaxial cables. The trigger is formed by a coincidence of any neighbouring OMs within a section (thresholds 0.5 and 3 p.e.). Data from the BEG (waveform for each triggered channel) are transmitted via an Ethernet connection (10 Mbit DSL-modem) to the underwater micro-PC of the cluster. The cluster DAQ centre provides intersection time synchronization, on-line data selection, and communication to shore through an optical cable.

\section{Prototype strings}

The operation of prototype strings in 2009 and 2010 allows a first assessment of the DAQ performance. The prototype string 2009 consists of 12 optical modules with six photomultipliers R8055 and six XP1807. In April 2010, a prototype of the GVD string with 8 PMTs R7081HQE and 4 PMTs R8055 was installed in Lake Baikal.

The accuracy with which the system can determine the arrival time of a photon at the photocathode has been determined from the LED flashers, laser calibration source, and cosmic ray muons. The first step of data analysis was a study of the accuracy of pulse time measurement with delayed pulses produced by the LED flasher [7]. The arrival time of a photon is determined from the waveform data. We find the delay values measured with 12 channels of the string to be close to the value that was installed on the LED flasher: the average deviation is about $1 \mathrm{~ns}$. The RMS of deviations is less than 2 ns. This value characterizes the time

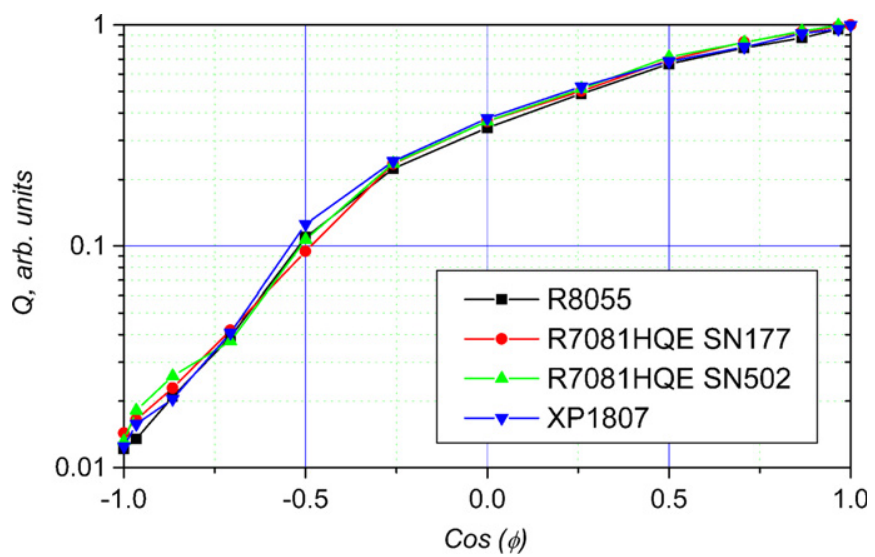

Fig. 1. Angular acceptance of OMs with various PMT types. resolution of the measuring channels. More detailed studies of time accuracy were performed with a laser source located at a distance of about $100 \mathrm{~m}$ from the prototype string. Time differences between pairs of channels measured for laser pulses are in good agreement with the expected values. The average deviation is about 2 ns. The flux of down-going cosmic ray muons enables testing of timing performance under the same conditions as those of actual data-taking. The muon analysis was performed for OMs with up-ward looking PMTs. Possible uncertainties of the OM angular acceptance at large angles do not influence the results of the OM response simulation in this case. Time differences between muon pulses detected with different channels were studied and compared with the simulation of the string response to the atmospheric muon flux [8]. The distribution of time difference between the optical modules along the string is in good agreement with the calculation. The difference between the peak positions of experimental and calculated distributions is about $2 \mathrm{~ns}$. This value characterizes the precision of the time measuring

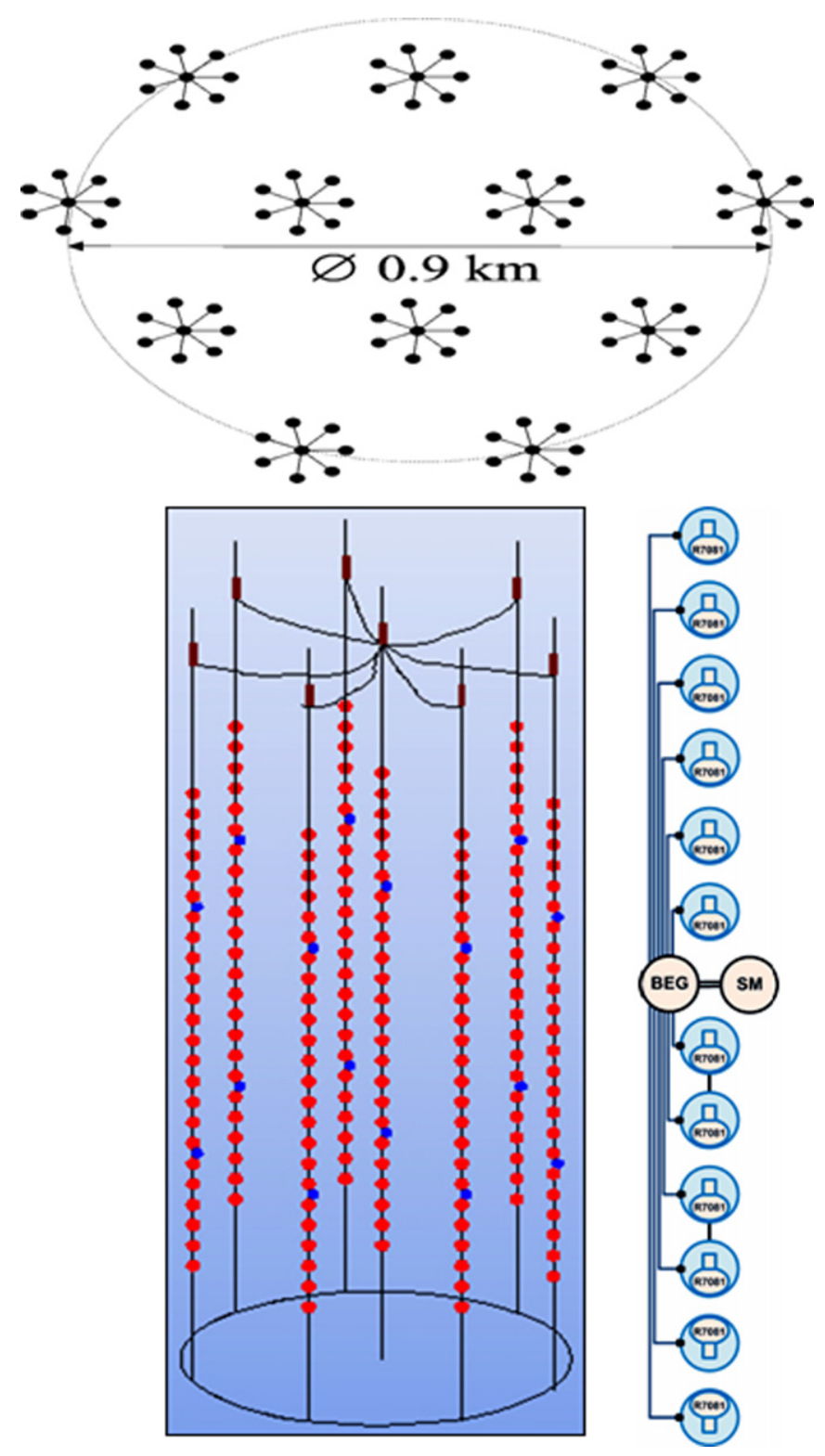

Fig. 2. GVD design: GVD top view (12 clusters), schematic view of a cluster ( 8 strings with 2 sections each), and a section of OMs (12 OMs with PMT R7081HQE). 


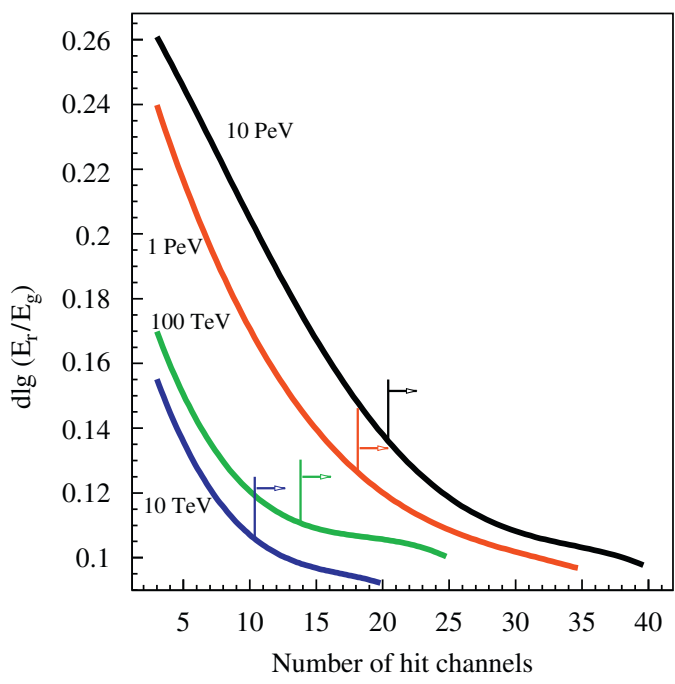

Fig. 3. Dependence of cascade energy resolution (RMS of $\log \left(E_{\mathrm{r}} / E_{\mathrm{g}}\right)$ on multiplicity of hit channels for different energies.

system, including both accuracy of time shift calibration and time resolution of the channels.

To estimate reconstruction efficiency and GVD effective area it is necessary to know the angular acceptance of the OM. The results of angular acceptance measurements are presented in Fig. 1 for OMs with various PMT types.

\section{Preliminary results of GVD optimization}

The objective of the GVD design optimization is to provide a maximum cascade detection volume with the condition that also high-energy muons are effectively registered. The present calculation was performed for 96 strings. Each string consists of two sections of OMs with PMT R7081HQE. Basic parameters for optimization were vertical distance between OMs $(Z)$, distance between string and cluster centre $(R)$, and distance between cluster centres $(H)$. Coincidences of any neighbouring OMs on a string (thresholds 0.5 and 3 p.e.) were used as a trigger condition.

An optimum for cascade detection volume and muon detection area was obtained for $H=300 \mathrm{~m}, R=60 \mathrm{~m}$, and $Z=15 \mathrm{~m}$. The optimized configuration of GVD is shown in Fig. 2. It comprises a total of 2304 OMs, arranged on 96 strings with 24 OMs each, and an instrumented length of $345 \mathrm{~m}$.

The detection area of this configuration for muons with energy from $10 \mathrm{TeV}$ up to $1 \mathrm{PeV}$ is $0.2-0.5 \mathrm{~km}^{2}$ with reconstruction error $0.5-\mathrm{l}^{\circ}$. The detection volume for cascades is $0.3-0.8 \mathrm{~km}^{3}$ with reconstruction error $3-7^{\circ}$ (cascade energy being $50 \mathrm{TeV}-1000 \mathrm{PeV}$ ). The efficiency of cascade parameters' reconstruction procedure depends on the number of hit OMs in an event. The accuracy of the cascade energy reconstruction is shown in Fig. 3.

The right-hand part of each distribution relative to the vertical line corresponds to $70 \%$ of total number of selected events. $E_{\mathrm{g}}$ and $E_{\mathrm{r}}$ are the generated and reconstructed cascade energies, respectively. For $70 \%$ of the events the accuracy of energy reconstruction $\log \left(E_{\mathrm{r}} / E_{\mathrm{g}}\right)$ is from about 0.09 up to 0.14 . Distributions of mismatch angles between generated and reconstructed cascade directions weakly depend on cascade energy. Fig. 4 shows the dependence of median value of mismatch angle distributions with fixed number of hit channels on hit channel multiplicity. For $70 \%$ of the events, the angular resolution is about $3-7^{\circ}$ for the energy range $10 \mathrm{TeV}-10 \mathrm{PeV}$.

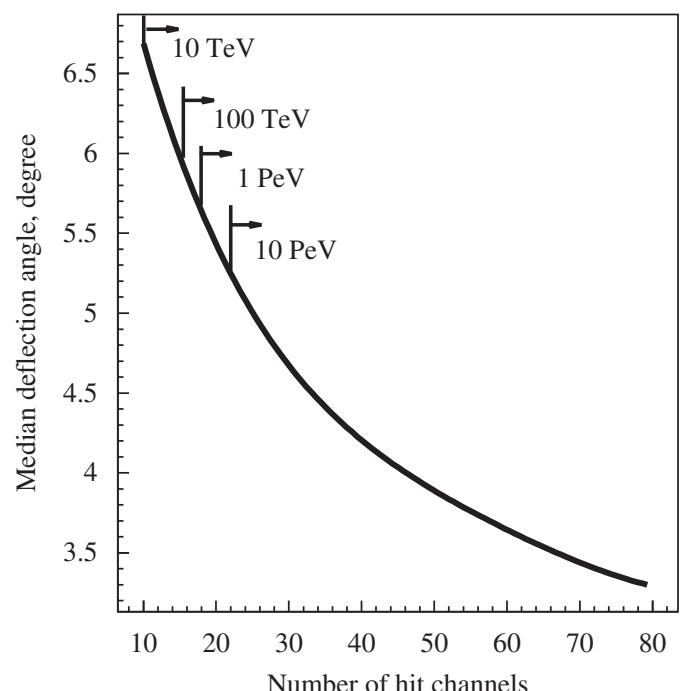

Fig. 4. Dependence of angular resolution of the cascade direction on multiplicity of hit channels.

\section{Conclusion}

The objective of the Baikal Project is the creation of a kilometer-scale high-energy neutrino observatory: the Gigaton Volume Detector (GVD) in Lake Baikal. Basic elements of the GVD DAQ (new optical modules with PMTs of various types, FADC readout units, underwater communication systems) were investigated and tested in situ with prototype strings in 2008-2010. Measurements with a LED flasher, a calibration laser, and muons allow one to estimate the time accuracy of the GVD measuring system at the level of about 2 ns. On the basis of the studies of the prototype string and optical module parameters the optimization of the GVD configuration was performed. The detection area of this configuration for muons above $10 \mathrm{TeV}$, with a reconstruction error of $0.5-1^{\circ}$, is $0.2-0.5 \mathrm{~km}^{2}$. The detection volume for cascades above $50 \mathrm{TeV}$, with a reconstruction error of $3-7^{\circ}$, is $0.3-0.8 \mathrm{~km}^{3}$. The technical design report for the GVD Baikal neutrino telescope will be finished in 2011 .

\section{Acknowledgments}

This work was supported in part by the Russian Ministry of Education and Science (GK 02.740.11.0018, GK 02.518.11.7158, P1242, P2504, P133, P878, P1146, RNP2.2.1.1/1483, RNP2.1.1/ 1539, RNP2.2.1.1/5901, REC "BAIKAL”), by the German Ministry of Education and Research, and by the Russian Foundation for Basic Research (Grant nos. 08-02-00432, 08-02-00198, 09-02-10001, 09-02-00623, 09-02-12295, and 10-02-10007).

\section{References}

[1] A. Avrorin, et al., in: Proceedings of the 30th ICRC Conference, Merida, Mexico 2007, arXiv:0710.3063.

[2] I. Belolaptikov, et al., Astropart. Phys. 7 (1997) 263.

[3] V. Aynutdinov, et al., Nucl. Instr. and Meth. A 588 (2008) 99.

[4] R. Bagduev, et al., Nucl. Instr. and Meth. A 420 (1999) ) 138.

[5] A. Avrorin, et al., Astron. Lett. 35 (2009) 651.

[6] V. Aynutdinov, et al., Nucl. Instr. and Meth. A 567 (2006) 433.

[7] V. Aynutdinov, et al., Nucl. Instr. and Meth. A 602 (2009) 227.

[8] A. Avrorin et al., Nucl. Instr. and Meth. A (2010) doi:10.1016/j.nima.2010.06.209. 